\title{
LES INSECTES AQUATIQUES
}

\author{
par H. BERTRAND \\ Docteur ès sciences, chargé du Laboratoire d'Éntomologie \\ à la Station centrale d'Hydrobiologie appliquée
}

Connaissance et ètude des insectes aquatiques offrent un intérêt économique qui n'est pas moindre que celui offert par les formes terrestres : pêche, pisciculture, limnologie théorique et appliquée non moins qu'hygiène publique et mẻdecine ont constamment à s'occuper de ces animaux qui, par ailleurs, par l'infinie variété de leurs formes comme de leurs mours, retiennent l'attention aussi bien du systématicien que du biologiste, et même du simple curieux des choses de la nature.

Nous ne nous proposons pas ici de procéder à une étude même élémentaire des insectes aquatiques, non plus de présenter en tableaux sypnotiques les éléments de détermination des insectes de nos eaux douces. En effet, étant donné le nombre des insectes, et la nécessité de pousser de tels tableaux au moins jusqu'aux coupes génériques, ceci déborderait à tous points de vue le cadre du Bulletin.

Nous nous bornerons donc à fournir au lecteur, un aperçu des formes aquatiques du groupe des insectes, de leurs caractéristiques et particularités morphologiques et biologiques, bien souvent fort curieuses, en indiquant par la même occasion, la place que les insectes aquatiques occupent dans le cadre de l'entomologie française et les plus importants des ouvrages et publications en langue française pouvant aider à leur identification précise. $A$ ce dernier point de vue, signalons tout de suite qu'il n'existe qu'un seul ouvrage — d'ailleurs inachevé et épuisé et non réédité - traitant de l'ensemble des insectes aquatiques d'Europe, c'est le Tome I du traité bien connu "Les Larves et Nymphes des Insectes aquatiques d'Europe " publié sous la direction de l'entomologiste belge Rousseau.

Cet ouvrage, ne concernant il est vrai, que les premiers états, intéresse les insectes paurométaboles (Hémiptères), hémimétaboles (Odonates, Plécoptères, Ephéméroptères), une partie des métaboles (Mégaloptères et Planipennes), c'est-à-dire une fraction déjà très importante des insectes aquatiques. Restent, sans parler des Coléoptères, souvent mal connus à l'état larvaire ou nymphal, le groupe immense des Diptères et les quelques Lépidoptères et Hyménoptères aquatiques. On trouvera seu-

(1) Pour les lecteurs peu familiarisés avec l'entomologie, nous rappelons que les termes " paurométabole" et " hémimetabole" s'emploient pour les insectes à métamorphoses faibles ou incomplètes, par opposition au terme " métabole" qu'on emploie pour les insectes à métamorphoses complètes. 
lement quelques notions élémentaires dans des publications de LÉGER, Brocher, Lestage, Germain.

On sait que l'embranchement des Insectes se divise en dẻux groupes principaux : les Insectes sans ailes ou Aptèrygotes et les Insectes ailés ou Ptérygotes, étant entendu que ce n'est pas seulement l'absence d'ailes, mais un ensemble de caractères, qui distinguent les uns des autres ces Insectes; car bien souvent des Ptérygotes ont des ailes réduites, sinon absentes; les Puces (Aphaniptères), par exemple, sont des Ptérygotes.

\section{I. - APTÉRYGOTES}

L'Aptérygote le plus connu est sans doute le curieux " Poisson d'argent ", cette bestiole gris perle, munie de trois longs filets caudaux (cerques) que l'on voit souvent évoluer dans nos habitations, particulièrement

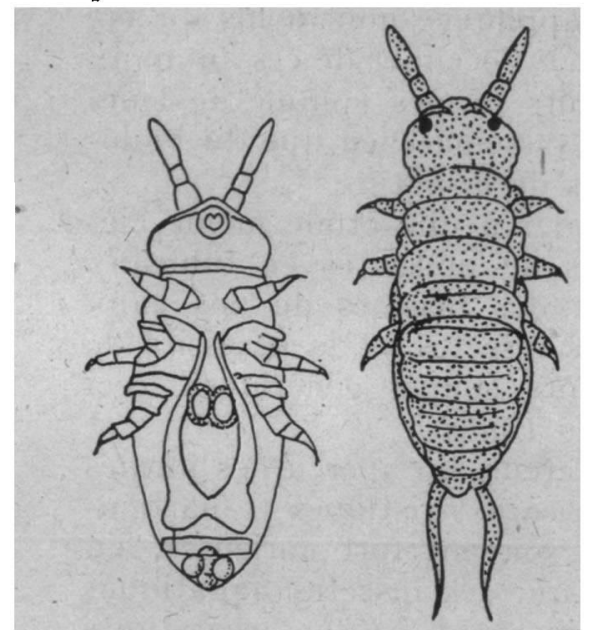

Podura aquatica. $\rightarrow$ A gaucle: face ventrale inontrant la furca repliee (d'après Brocher). parmi les vieux papiers. Cet Insecte appartient à l'ordre des Thyśanoures, caractérisé notamment par la présence de rudiments de membres abdominaux. Aptérygotes aussi, sont les minuscules Collemboles, par exemple ces Podures de teinte bistre que l'on voit souvent en nombre considérable aussi bien sur les flaques d'eau salée le long de nos côtes que sur le bord des mares, voire les ornières inondées des chemins. Au même groupe appartient encore la "Puce des glaciers", qui vit sur les neiges.

La plupart de ces insectes surtout amis de l'humidité, cachés souvent dans l'humus ou assez profondément dans le sol, sont à peine aquatiques; on désigne comme tels ceux qui vivent en effet "à la surface de l'eau " et leur corps, non mouillable, n'y pénétrant pas. Les Collemboles sont dépourvus d'yeux composés et surtout remarquables par la présence d'un membre impair, en fourche, la furca, sorte de ressort replié sous le ventre qui leur permet d'effectuer des bonds étendus. Comme tous lesAptérygotes, ils ne subissent pas de métamorphoses, du moins bien apparentes (Amétaboles).

\section{II. - Ptérygotes}

\section{Les Hémiptères.}

Comme on sait bien sensibles, mais graduelles, et en tout cas sans changement accusé de facies, ni arrêt de leur activité, se présentent les métamorphoses chez les Criquets, les Sauterelles, les Punaises ou Hémiptères. Les derniers de ces "Paurométaboles " fournissent un contingent assez important à la faune des eaux douces; il en est même de marins.

Parmi les " Punaises d'eau », il en est tout un groupe, caractérisé par 


\section{Hémiptères}

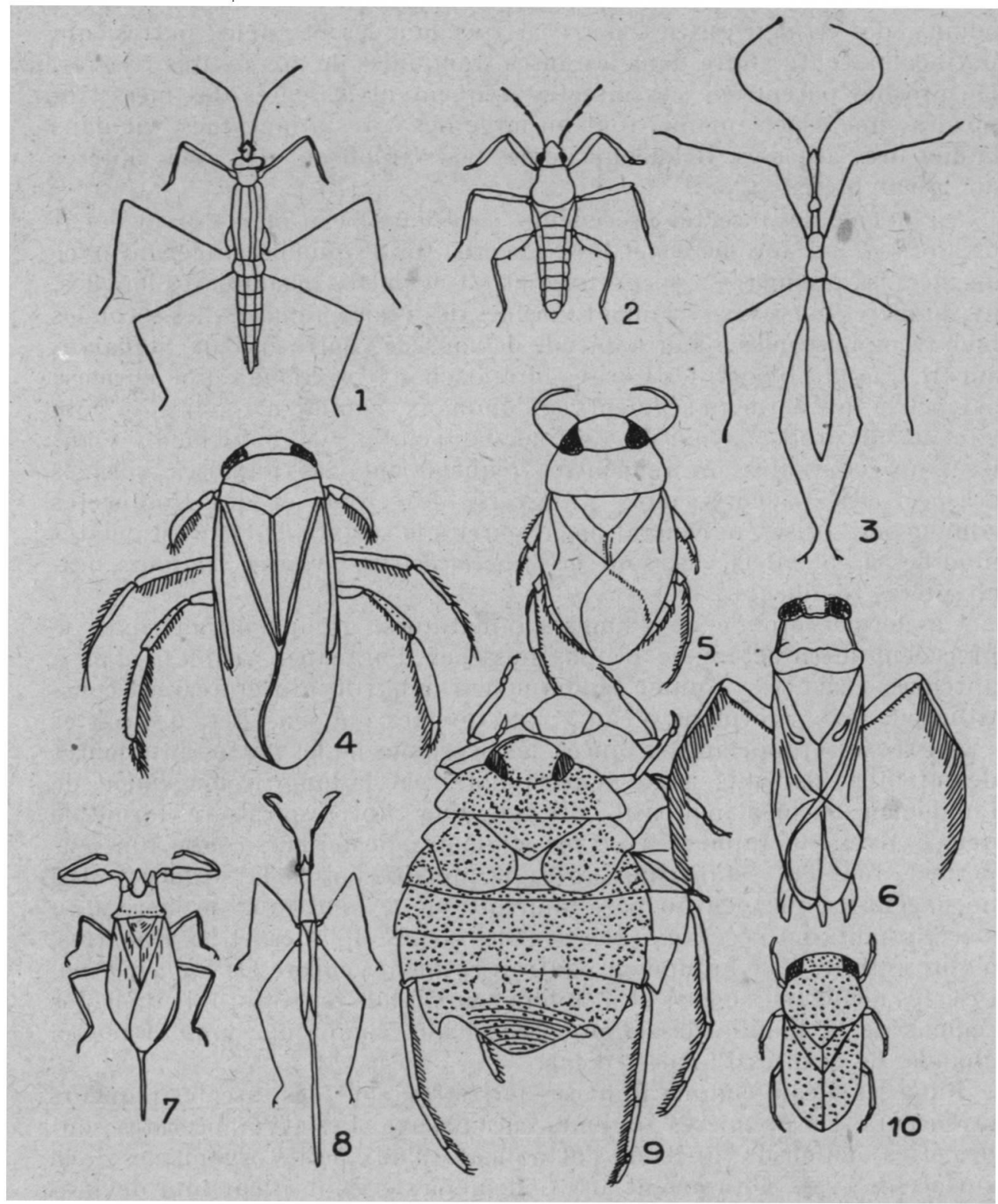

1, Gerris najas de Geer; 2, Velia cumens F. (d'après Jensen Hanup) ; 3, Hydrometra stagnarum L. (d'aj)rès Kunlgatz); 4, Corixa Geoffroyi Leach (d'après Minlu); 5, Naucoris cimicoides L. (d'après Jersen HaAnup); 6 , Notonecta glauca L. (d'après Kunlgatz); 7, Nepa; 8, Ranatra (d'après Nezd нaм); 9, Aphelocheirus aestivalis F., insectes accouplés (d'après LArSeN); 10, Plea minutissima F. (d'aprés JENSEN HAARUP). 
des antennes bien visibles, nues (Gymnocérates) qui, comme les Collemboles, vivent exclusivement à la surface des eaux; ce sont les filiformes Hydromètres qui marchent sur l'eau, les plus agiles Vélies, qui courent comme des Araignées, les Gerris à très longues et grêles pattes, qui patinent à toute allure dans les anses tranquilles de nos petites rivières. De proches parents de ces insectes fréquentent les côtes des mers tropicales; une espèce même, tout au large des côtes atlantiques, vit dans la mer des Sargasses. Beaucoup de ces insectes offrent des races aptères ou brachyptères.

A l'inverse des insectes précédents, c'est dans l'eau même qu'évoluent les Corises, les Notonectes et les Naucoris, toutes punaises nageant avec aisance, les premières à corps assez étroit et pattes postérieures longues, les dernières massives, vraiment voisines des vraies punaises, les secondes bien reconnaissables à leur habitude de nager le ventre en l'air. Signalons encore que la plupart des Corises offrent chez le sexe mâle une curieuse asymétrie des derniers segments abdominaux, comme atrophiés du cóté gauche ou droit, asymétrie destinée à faciliter l'accouplement. Dans les eaux courantes on rencontre fréquemment des punaises voisines d'aspect des Naucoris, mais à l'inverse de ceux-ci et des Notonectes comme des Corises, ne venant pas respirer à la surface de l'eau (stigmates modifiés à cet effet) : ce sont les A phelocheirus, presque toujours brachyptères d'ailleurs.

Un dernier groupe de Punaises aquatiques, groupe offrant avec le précédent le caractère de ne pas posséder d'antennes visibles, mais à antennes "cachées " comme l'indique leur nom (Cryptocérates) est constitué par les "Scorpions d'eau"; ces insectes ont, en effet, des pattes "ravisseuses " rappelant les pinces des Scorpions ou les pattes antérieures des Mantes; ils sont encore remarquables par le long prolongement de l'abdomen, ici bien inoffensif; il s'agit d'un tube respiratoire, formé de deux valves, en rapport avec les stigmates postérieurs, tube qui leur permet, tout en restant immergés, de prendre l'air ả la surface; aussi ne nagent-ils que peu ou pas, rampant sur le fond près de la rive ou s'accrochant aux végétaux : tels sont les Nèpes, aplaties, et les Ranatres, à corps étroit. A ce groupe on pourrait rattacher encore les Sphærodema, insectes meilleurs nageurs et à siphon court, seuls représentants dans nos régions de la famille des Belostomatidæ qui comptent, dans la région chaude, de géantes Punaises d'eau.

Punaises d'eau, comme Punaises terrestres, sont des insectes piqueurs et suceurs, les premières toujours carnassières. Les Gymnocérates sont des êtres inoffensifs car ils ne s'attaquent qu'aux petits organismes de la surface de l'eau, notamment aux Collemboles et sont à leur tour dévorés par les poissons ou autres prédateurs.

Par contre, on ne peut en dire autant des Cryptocérates, plus particulièrement des Corises et surtout Notonectes. Ces voraces sont nuisibles et s'attaquent à toutes sortes d'animaux, poissons compris; non dangereux pour l'homme, on devra toutefois éviter de les manipuler sans précautions, car leur piquire est réellement douloureuse.

Les Punaises d'eau pondent leurs œufs au voisinage de l'eau, sur les objets immergés ou bien encore les enfoncent à l'intérieur des tiges et 
feuilles des végétaux aquatiques, certaines même les transportent sur le dos des mâles (Belostomatidæx); ces ceufs offrent les aspects les plus variés : globuleux et arrondis, pédicellés, ou encore avec des prolongements en nombre variable (Nèpes, Ranatres); ajoutons que, souvent, ces oufs sont parasités par de minuscules Hyménoptères, et que ceux de certaines espèces (Corises) sont recueillis et consommés par l'homme (Mexique, Égypte).

Les premiers états des Hémiptères aquatiques sont étudiés dans le traité de Rousseau. Les jeunes différant comme indiqué ci-dessus relativement peu de formes marginales - ils en conservent les caractères essentiels. En l'absence d'un ouvrage spécial pour l'identification des espèces de nos régions assez bien connues, on consultera avec profit diverses publications de R. Porsson.

\section{Les Odonates ou Libellules.}

Les Odonates ou Libellules sont parmi les Insectes aquatiques les plus connus, les imagos attirant l'attention par leurs évolutions et parfois la richesse de coloris du corps, plus rarement des ailes. Il existe deux sous-ordres principaux (les deux seuls connus de nos régions) : les Anisoptères ou Libellules proprement dites et les Zygoptères ou Demoiselles.

Les Odonates ont une grosse tête ou plutôt de gros yeux, parfois plus ou moins contigus ; ce sont, d'autre part, des carnassiers voraces à pièces buccales du type broyeur. Leurs ailes généralement transparentes, parfois colorées par places ont un aspect " réticulé " très caractéristique; ce dense réseau, disposition primitive, masque dans une certaine mesure les nervures principales longitudinales ou transversales dont la disposition a une grande importance systématique. Le thorax, auquel la tête s'attache par une grêle articulation, a une structure particulière; Ies flancs (ou pleures) à pièces très allongées et obliques; les pattes reportées très en avant; les ailes insérées, au contraire, en arrière. L'abdomen toujours assez long, quelquefois plat, est parfois très grêle (chez les Demoiselles); sa structure varie selon le sexe. Chez les mâles, il existe toujours au bout de l'abdomen une paire d'appendices sapérieurs formant souvent des pinces ou forceps, également un appendice inférieur impair au-dessus de l'anus (Anisoptères), des appendices pairs au-dessous (Zygoptères). Chez les femelles, les appendices précédents sont plus ou moins réduits, quelquefois, cependant, ,les appendices inférieurs forment de longs rubans ; de plus, les femelles possèdent des valves formant un appareil de ponte oviscapte plus ou moins développé. Ajoutons encore que, chez les mâles, il existe constamment un appareil complexe, appareil de copulation, situé vers la base de l'abdomen, à la face ventrale du deuxième segment. Les deux sexes diffèrent souvent par la coloration, ce qui est le cas chez beaucoup de Demoiselles, soit par le corps, soit par les ailes (cas des Demoiselles à ailes bleues, les Calopteryx (1), dont les femelles ont les ailes brunes). L'accouplement des Odonates, qu'il est si facile d'observer dans

(1) Agrion dans la nomenclature actuelle. 
la nature, constitue une des singularités de ces insectes. Le mâle saisit la femelle à l'aide de ses pinces et appendices anaux soit entre tête et thorax, soit au niveau du prothorax et les deux conjoints volent ainsi l'un derrière l'autre. Mais le rapprochement des sexes ainsi réalisé, la fécondation mème demande une manœuvre singulière. Tout d'abord les orifices sexuels du mâle étant vers le bout de l'abdomen et l'appareil de copulation tout en avant, il est nécessaire que l'animal procède préalablement à la charge en sperme de l'appareil copulateur par déplacement de l'abdomen; ensuite que la femelle, dont les orifices sexuels sont, eux aussi, postérieurs, vienne, en reployant son abdomen en boule, les placer au contact de l'appareil copulateur.

La ponte s'effectue selon divers modes. Quelquefois les femelles se bornent à déposer ou laisser tomber leurs oufs près de l'eau, à la surface, ou tout au plus à les enfoncer dans la vase, mais dans d'autres cas pratiquant des entailles dans les arbrisseaux ou plantes aquatiques, elles les cachent complètement au cour des tissus végétaux; c'est ce que font les Eeschnidæ (Anisoptères), encore les Zygoptères du genre Lestes provoquant des sortes de "galles" sur les Saules (PIERRE) ou les Platycnemis du même groupe que nous avons vu nous-même pondre dans les tiges de Renoncule. De l'œuf des Odonates sort un organisme assez singulier, la" " pronymphe ", stade tout transitoire libérant presque aussitôt une nymphe active, insecte aquatique assez différent de la Libellule, d'abord sans ailes, mais déjà muni d'yeux composés et de longues pattes; les Odonates sont, en effet, des Hémimétaboles. Chacun connaît ces nymphes d'Odonates, animaux carnassiers dont la lèvre inférieure a double articulation, s'élargit en une palette armée de crochets appliquée au repos sur la face et la bouche, d'où son nom de masque. Nymphes de Libellules et de Demoiselles ne sont pas semblables; les premières ont au bout de l'abdomen des pointes qui s'accolent en "pyramide" au repos, les secondes, des lamelles ou branchies en partie au moins triédriques, ou toutes aplaties, au nombre de trois. Ajoutons encore que, tandis que les nymphes des Demoiselles respirent en principe par leurs lamelles, celles des Libellules pratiquent la respiration intestinale, plus précisément rectale, car la paroi de l'intestin postérieur offre un système complexe de plis recevant des rameaux et ramuscules de l'appareil trachéen. Disons encore que la chasse brusque de l'eau contenue dans le rectum constitue chez les nymphes un mode de propulsion rapide, rappelant le mécanisme utilisé par la Seiche et les Mollusques céphalopodes.

Il est relativement facile de distinguer entre, elles les nymphes des divers Odonates, tout au moins celles des Libellules ou Anisoptères; d'ailleurs ces dernières ont tantôt le corps allongé, tantôt le corpṣ trapu, court et se tenant soit sur le fond, soit même enfoncées dans la vase sont dites fouisseuses, les unes à masque plat (Gomphidæ) d'autres à à masque concave à dentelures régulières (Libellulidæ) ou non (Cordelugasteridæ2). Parmi les Zygoptères, on reconnait aisément les nymphes des Calopteryx aux branchies prismatiques, celles des Conagrionidæ aux ramifications obliques des trachées, les mèmes à angle droit chez les Lestidær.

A l'état de nymphes, les Odonates habitent surtout les eaux stagnantes, 


\section{Odonates}

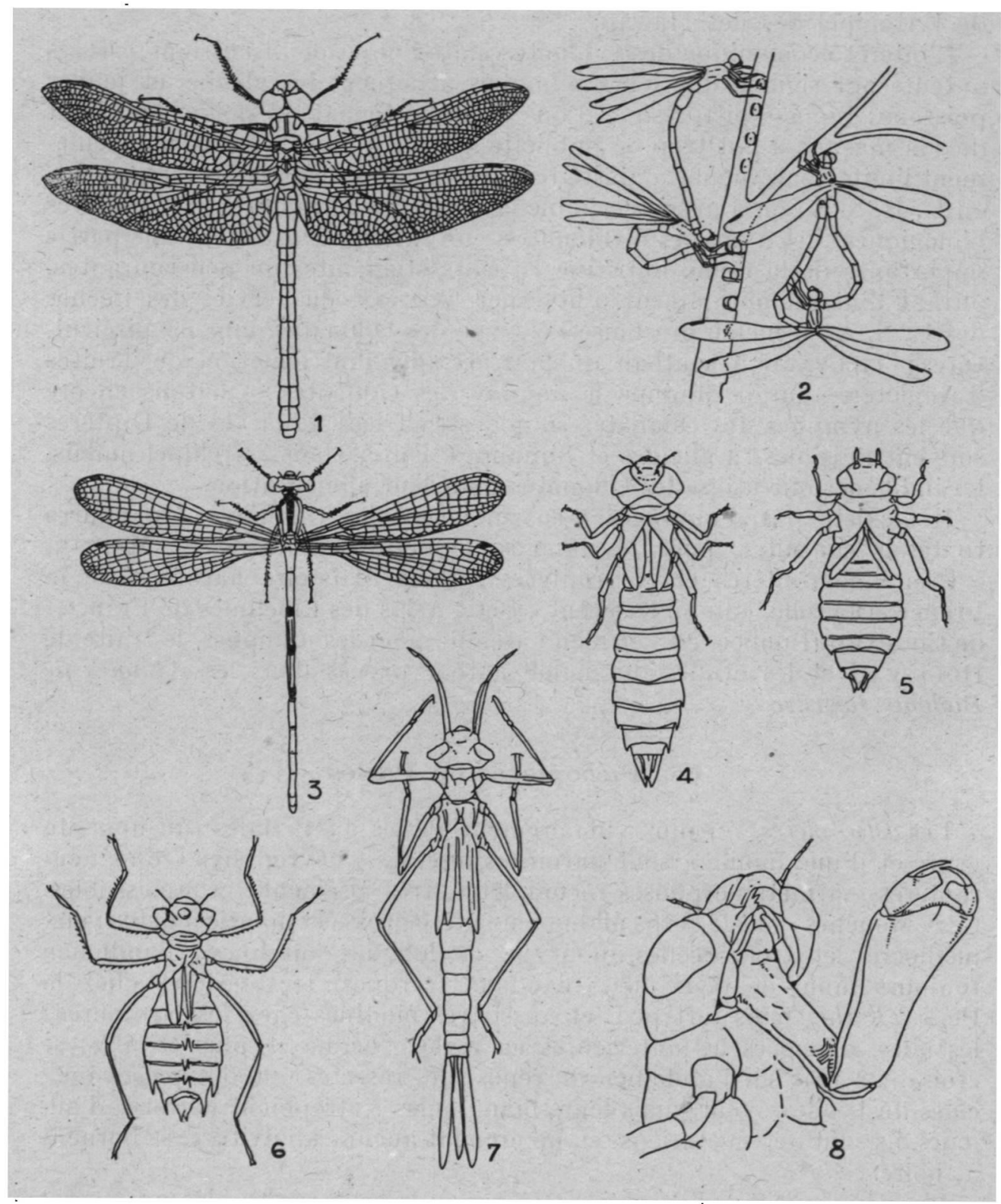

1, Macromia magnifica Mcl. (d'après KBNend); 2, Arehilestes californica Mcl.., à gauche femelle pondant, à droite insectes accouplés (d’après KENNED廿); 3, Ischnura perparva Sélys (d'après KBNNEDY) ; 4, larve d'Aeschna; 5, larve d'Ophiogomphus (d'après NegBaAu), 6, larve d'Orthetrum (d'après houssaul); J, larve d'Agrion [Calopteryx auct] (d'après Caopard); 8, tête d'une larve d'Anax avec masque en demi-flexion (d'après Wrara). 
parfois les eaux courantes, rarement les torrents (hors d'Europe) ; il en est même de subterrestres vivant dans les régions tropicales à l'aisselle des feuilles, dans le terreau des Fougères (notamment dans les montagnes de l'Archipel des îles Hawaï).

L'intérêt économique des Odonates a été l'objet de discussions. Divers auteurs ont signalé que leurs nymphes attaquent les alevins et jeunes poissons; l'image pittoresque d'une nymphe tenant un poisson au bout de son masque a fait trop de publicité autour d'un rôle nocif, non seulement limité, mais même contesté par divers naturalistes. Plus récemment, en U. R. S. S., on a aussi incriminé ces insectes de rompre des équilibres biologiques. Les nymphes d'Odonates, en tout cas, constituent une partie importante de la faune nutritive en eaux stagnantes ou peu courantes, offrant l'intérêt notamment d'hiverner. Wılson, du Service des Pêches des U. S. A., a même préconisé l'élevage des Odonates dans les piscicultures; Trluyard a soutenu qu'en Australie, l'introduction de Truites d'Angleterre aurait diminué le nombre des Odonates. Ajoutons encore que les nymphes des Odonates sont destructrices de larves de Diptères souvent nuisibles : Culicides et Simulides. Enfin, dans l'Archipel malais, les indigènes ont utilisé les Odonates pour leur alimentation.

Il n'existe pas d'ouvrages d'ensemble sur les Odonates. On pourra toutefois consulter, pour les imagos, le petit volume de R. MARTin, "Pseudonévroptères et Névroptères " dans l'Histoire naturelle de la France (Deyrolle, éd.) ou le récent "Petit Atlas des Libellules de France " de Chopard (Boubée, éd.) et bien entendu, pour les nymphes, le traité de Rousseau et les études du même auteur parues dans les Annales de Biologie lacustre.

\section{Les Plécoptères ou Perles}

Les Plécoptères, ou plus vulgairement Perles ou Perlides (du nom du genre et d'une famille), sont parmi les insectes " névroptères" ou " neuroptères " à métamorphoses incomplètes, très aisément reconnaissables. Ces "Mouches" ont le corps allongé, une tête grosse et aplatie à deux yeux médiocres et trois ocelles, pourvue de longues antennes; l'abdomen toujours muni de deux filets caudaux (cerques) très visibles chez la Perle (Perla) mais fort peu, et aussi très modifiés chez les Nemoures; les ailes, allongées les antérieures en règle générale et plus étroites, se croisant à plat sur l'abdomen au repos. Ces insectes ont des pièces buccales du type broyeur, mais leurs mandibules s'atrophient parfois; d'ailJeurs, ils sont ou végétariens ou, ne prenant aucune nourriture, se bornent a boire.

L'accouplement a lieu à terre et les oufs sont rassemblés en masses ovigères, transportées par les femelles.

Les nymphes de Plécoptères, à tête aplatie et corps allongé portent toujours deux longs filets caudaux (cerques) à l'extrémité de l'abdomen, leur corps ressemble assez à celui des insectes parfaits; leurs antennes sont bien développées.

Souvent aplaties, ces nymphes, surtout celles de quelques grosses formes (Perlida, Perlodidx) n'offrent qu'une ressemblance toute super- 
ficielle avec les nymphes des Éphémères ou Éphémêroptères; au moIns chez les formes de nos régions, elles s'en différencient par l'absence de branchies abdominales, latérales ou dorsales et toujours, à la différence des nymphes des Éphémères, elles possèdent des pattes munies de deux griffes. Beaucoup de ces nymphes ne possèdent pas de branchies et ont une respiration cutanée; quand les branchies sont présentes, ce sont des houppes de filaments ou filaments isolés insérés sous le thorax, au cou, à la base des pattes, auprès de l'anus.

Les nymphes des Plécoptères sont assez homogènes d'aspect. Toutefois on reconnâtra celles des Leuctra à leur corps très grêle; celles des Nemoures, à fourreaux alaires divergents, sont bien connues sous le nom de "Grillons d'eau ", les nymphes des Trniopteryx (Voilette) ont de

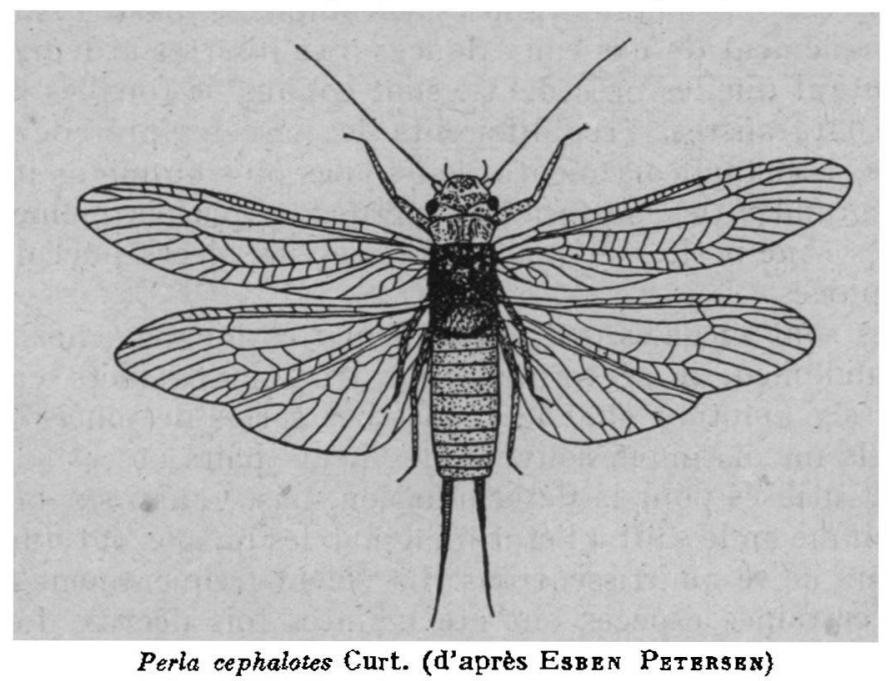

curieuses épines le long du dos, sans compter les grosses nymphes des Perlidæ et Perlodidx. Parvenues au terme de leur croissance, les nymphes, généralement de nuit, abordent quelques supports émergeant et, comme chez les Odonates, la peau se fend pour laisser échapper l'insecte parfait. Mais ici intervient souvent un curieux phénomène, la nymphe rejetant d'abord le revêtement de la partie antérieure du tube digestif qui sert d' " ancre " à laquelle l'insecte s'attachera.

Sauf les grosses formes, à régime mixte, les nymphes des Plécoptères sont végétariennes, microphages, se nourrissant des couvertures biologiques. Comme les insectes parfaits, qui servent de "mouches " pour les Salmonidés, les nymphes sont très recherchées par les poissons et LESTAGE a noté particulièrement leur valeur nutritive.

Les nymphes des Plécoptères habitent rarement les eaux stagnantes, mais il en est qui se contentent des rivières, beaucoup abondent dans les torrents de montagne, quelques-unes vivant sur les rochers mouillés (faune hygropétrique de ThIENEMANN). D'une façon générale, ce sont des animaux recherchant les eaux pures et froides; aussi les rencontre$t$-on jusque dans les régions polaires et les hautes montagnes; nous avons nous-même recueilli des nymphes dans la zone alpine des Pyrénées et 
l'expédition anglaise de l'Everest a observé des Plécoptères vers 5.200 mètres sur le glacier de Rongbuk.

Il n'existe pas encore d'ouvrage récent et complet sur les Plécoptères de nos régions, tout au moins pour identifier les espèces.

Depuis longtemps, particulièrement dans la région pyrénéenne particulièrement riche, le Professeur Despax a découvert nombre d'espèces intéressantes et un prochain volume de la Faune de France rédigé par lui traitera des Plécoptères de notre pays.

\section{Les Éphéméroptères ou Éphémères.}

Les Ephéméroptères ou Éphémères constituent le troisième et dernier groupe d'insectes à métamorphoses incomplètes participant à l'état jeune au peuplement de nos eaux douces. Ces insectes et leurs nymphes, au moins autant que les précédents, sont connus de tous les pècheurs et de tous les naturalistes. Très différents des insectes précédents à l'état parfait on les reconnaît aisément à leurs ailes plus ou moins inégales, les antérieures grandes, les postérieures petites et parfois même absentes. Les antennes sont petites et peu visibles et les pièces buccales plus ou moins atrophiées.

Les pattes sont inégales, les antérieures très longues chez les mâles; quant à l'abdomen, il se termine par trois longs filets caudaux ou "cerques"; s'y ajoutent chez les mâles des sortes de pinces ou forceps entre lesquels on distingue souvent des pénis pairs et ces "genitalia" sont souvent utilisés pour la détermination, basée aussi sur la nervation des ailes. Comme on le sait, à l'état imaginal, les imagos ont une vie brève et par ailleurs ne se nourrissent pas. Ils volent fréquemment en essaims et ceux de certaines espèces ont été maintes fois décrits. Les Polymitarcis notamment forment de véritables nuées sur le bord des rivières et fleuves; ces essaims se constituent souvent a la fin du jour, et, la nuit venue, les insectes sont attirés par les lumières. Il nous souvient à ce propos d'avoir été témoin à la tombée de la nuit d'un curieux spectacle provoqué par ce "phototropisme ". Une haute colonne d'Éphémères (Polymitarcis virgo) s'était constituée à l'entrée d'un pont sous un globe électrique lorsqu'une automobile vint stationner à faible distance. Les phares de cette dernière ayant été allumés, le double faisceau lumineux vint à son tour frapper les insectes. Aussitôt un double courant d'Éphémères se détacha à angle droit. Tourbillonnant en folles spirales, les Polymitarcis vinrent bientôt former deux véritables tas devant la voiture.

L'accouplement a lieu au vol, le mâle saisissant la femellè précisément à l'aide de ses pattes antérieures. Quant à la ponte, comme chez les Plécoptères, elle s'effectue selon des modes variés, certaines femelles laissant tomber des œufs isolés mème en volant ou en touchant à peine la surface de l'eau; d'autres lâchant tout un paquet d'œufs qui se désagrège au sein du liquide, ou bien enfin pénètrant sous l'eau pour déposer les œufs sur des supports immergés.

Les nymphes des Éphéméroptères, toujours aquatiques, sont de forme et d'aspect très divers; certaines ont le corps cylindrique, d'autres sont 
aplaties mais elles ne peuvent en aucune manière être contondues avec celles des Perles ou Plécoptères.

La différence réside non tant dans la présence de trois filets caudaux au lieu de deux à l'extrémité de l'abdomen - le cerque médian peut, en effet, s'atrophier ou disparaître - que dans la présence de branchies latérales visibles sur les côtés ou la face dorsale des segments abdominaux, les pattes par ailleurs toujours terminées par une seule griffe, toujours deux chez les Plécoptères. On désigne d'ailleurs sous le nom de branchies aussi bien des expansions foliacées, à un seul ou plusieurs feuillets, que des filaments en " touffes" ressemblant à celles des nymphes de Plécoptères, le deuxième type s'associant souvent au premier et pouvant apparaître à la base même des mâchoires comme chez les curieuses nymphes des Oligoneuria; les branchies revêtent enfin un aspect "plumeux" chez les nym. phes bien connues des Éphémères proprement dites. Les lamelles des nymphes sont plus ou moins mobiles et provoquent des courants, facilitant la circulation $d u$ liquide et la respiration; chez les Éphémères, on constate aussi un mouvement très caractéristique

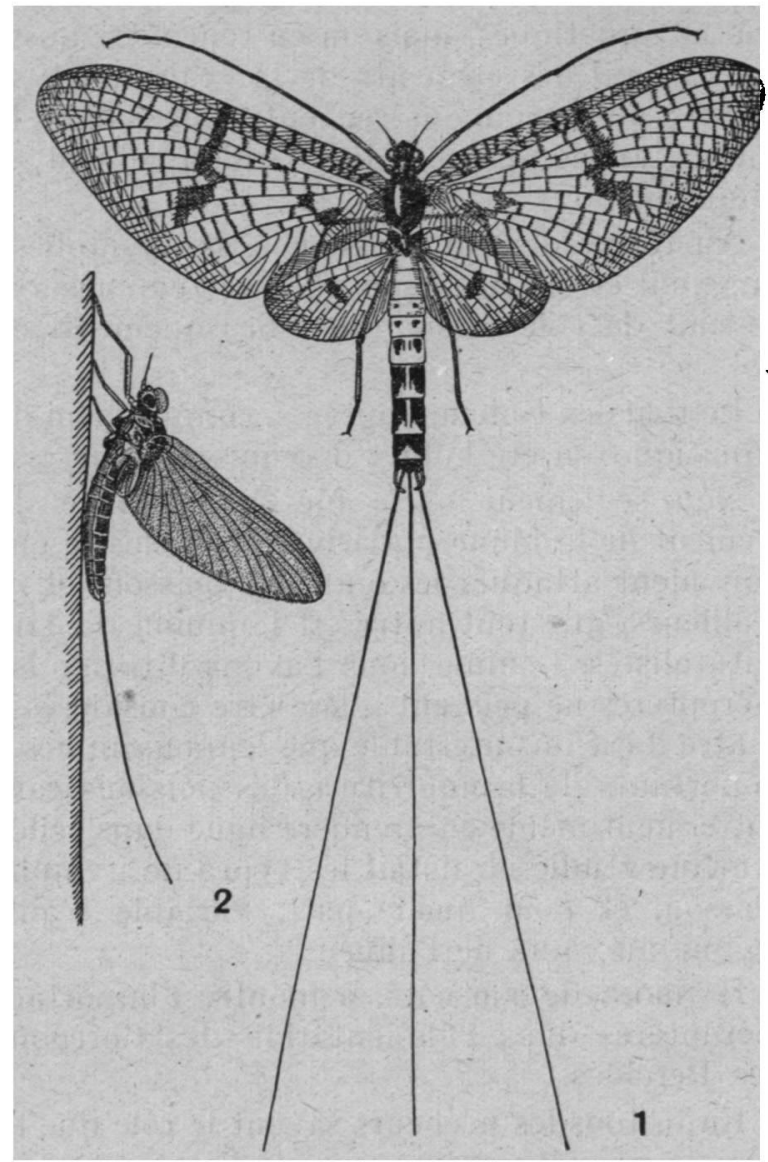

1, Ephemera danica Mull., mâle; 2, Bactie rhodani Píct., mâle (d'après Kínurss).

des branchies plumetıses; d'une façon générale, on a constaté que Ia mobilité des branchies différait selon le mode de vie des nymphes, diminuant chez les formes des eaux courantes riches en oxygène. A ce propos nous remarquerons que les nymphes des Éphémères à branchies mobiles se conservent bien dans de l'eau non renouvelée. On a ètabli ainsi un rapprochement entre la forme des larves et leur genre de vie; tout cela n'a rien d'absolu, mais il faut tout de même constater qu'à côté de formes de petite taille, non aplaties comme les nymphes des Bætis, on trouve dans les torrents les plus rapides des "pétricoles " aplaties qui, comme celles des Epeorus supportent les courants les plus forts comme cela a été expérimentalement démontré encore dernièrement par DoRIER et Vaillant. Certaines nymphes d'Éphéméroptères sont d'aspect singulier, 
notamment celles des Behningia de l'Europe orientale et de Sibérie et celles célèbres des Prosopistomes jadis prises pour des Crsutacés, étudiées notamment par VAYSSIËRE, et que nous avons eu nous-mème l'occasion de recueillir en Gironde. Les nymphes des Éphéméroptères ont un régime mixte ou plus souvent sont phytophages ou microphages, vivant aux dépens des couvertures biologiques; comme nous l'avons dit elles sont toutes aquatiques, mais on en rencontre aussi sur des rochers ruisselants constituant des éléments de la faune dite "hygropétrique" de ThieneMANN; nous .en avons rencontré ainsí dans les Pyrénées sur le bord des routes, en compagnie de "Grillons d'eau " (Nemuridés) et parfois de Simulies.

Au terme de leur croissance, les nymphes passent par un stade subimaginal et chacun a pu observer ce curieux spectacle de la "Mouche" cessant de voler, pour muer brusquement, et sans tarder reprendre son vol.

Le rôle des Éphéméroptères, comme celui d'ailleurs de bien des insectes aquatiques, a été l'objet de contestations.

Non seulement on a nié l'importance des Éphéméroptères comme élément de la faune nutritive, mais encore on a soutenu que les nymphes pouvaient attaquer les oufs des poissons et même les alevins. Ajoutons, d'ailleurs, que tout autre est l'opinion de Tillyard et de la plupart des naturalistes. Comme nous l'avons dit, par leur régime même, les Éphéméroptères ne peuvent guère être consiidérés comme des prédateurs; par contre il est incontestable que leurs nymphes constituent un des éléments importants de la nourriture des poissons carnassiers et, d'après VAvoN, entreraient même en première ligne dans celle de la Truite; STankovitch a même étudié en détail les types de nymphes attaquées par ce dernier poisson, et pour quelle part, variable d'ailleurs, elles entrent dans le régime au cours de l'année.

Ranson, de son côté, a montré l'importance des nymphes des Éphéméroptères dans l'alimentation des Corégones, et CLÉmens dans celle des Percidés.

Enfin tous les pêcheurs savent le rôle que les Éphémères jouent encore dans la nourriture du poisson à l'état de subimago et imago aux époques d'éclosion. Pour les nymphes, Lestage conclut lui-même en ces termes :

"Ces petites formes nageuses et phytophiles sont un aliment précieux pour l'alimentation des alevins; les larves fouisseuses constituent une part importante de la faune nutritive des petites rivières et ruisseaux; toute la série des formes pétricoles, particulièrement représentées dans les torrents rapides des montagnes et jusque dans les lacs froids de la région alpine, joue un rôle particulièrement important dans l'alimentation de la Truite."

On peut, grâce au traitẻ de Rousseau, reconnaître assez facilement les principaux genres d'Éphéméroptères de nos régions à l'état nymphal, encore qu'un des travaux les plus complets et mieux illustrés soit en langue allemande (SCHCENEMUND). Nous n'avons pas encore de travail d'ensemble sur ce groupe et la faune française paraît à l'heure actuelle incomplètement connue. LestaGe a donné jadis un catalogue des espèces 
Éphéméroptères et Plécoptères

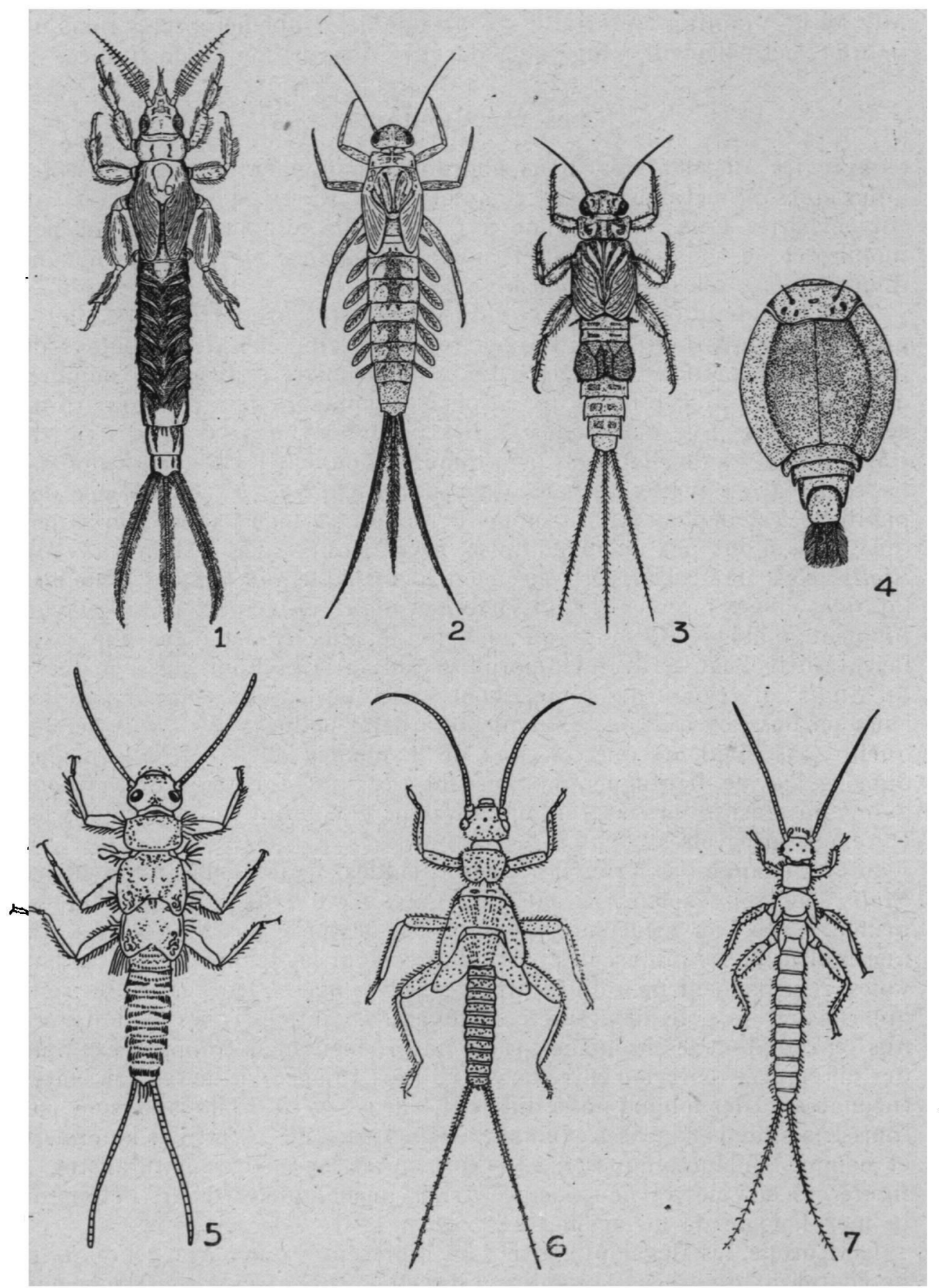

Ephéméroptères: 1, larve d'Ephemera ; 2, larve do Baetis ; 3, larve de Caenis (d'après Scrozngmuxd) ; 4, larve de Prosopistoma (d'après Vayssiḱne). Plécoptères : 5, larve de Perlidae (d'après NeEdax) ; 6, larve de Nemura; 7 , larve de Leuctra (d'apress Rousseau). 
françaises, mais des additions nombreuses doivent y' être faites, comme en témoignent les publications de divers auteurs, notamment de Mlle M. L. Verrier qui traite du groupe des Éphéméroptères dans un volume, actuellement rédigé et à paraître dans la Faune de France.

\section{Les Mégaloptères.}

Avec les Mégaloptères, nous abordons le premier groupe d'Insectes aquatiques à métamorphoses complètes. Chez eux et les insectes qui suivent, à la larve succède une nymphe vraie, organisme qui est peu mobile, et en tout cas ne subit aucune croissance et ne prend aucune nourriture : c'est la métamorphose typique.

Tout le monde connaît les Sialis, ces insectes brunâtres, aux ailes à grosses nervures noires, en toit au repos, comme chez les papillons de nuit. Dès le premier printemps on les rencontre, souvent en nombre, accrochés aux brins d'herbe ou parfois aux feuillages des arbres ou arbrisseaux sur la berge des étangs et des petites rivières; d'où le nom de "Mouches de saule" (Alder-Flies) que leur donnent les pêcheurs anglais. Le Sialis a des pièces buccales du type broyeur et, à la différence des papillons, est dépourvu de trompe, caractère partagé par les Phryganes qui, elles, n'ont pas de mandibules. Bien connue aussi est la larve du Sialis ; c'est une carnassière, aisément identifiable aux longues branchies latérales ciliées insérées sur un abdomen mou, lui-même terminé par un filament analogue. Ce n'est qu'au bout de plus de deux ans que cette larve quitte l'eau et se métamorphose sur la rive. Fouisseuse, la larve du Sialis affectionne les fonds sablovaseux ou vaseux, supportant des eaux parfois peu oxygénées, et mème à demi polluées. Le Sialis ne fréquente pas d'ailleurs que les eaux de la plaine; on le retrouve jusque dans les lacs de montagne, apparaissant alors plus tard en saison et nous avons pu ainsi observer sa nymphose dans le courant de juillet dans les Pyrénées ariégeoises.

Si l'on examine les brins d'herbe ou feuilles sur lesquelles reposent les Sialis, on trouve bien souvent un "gâteau " d'cufs cylindroconiques, brunâtres. Si l'on recueille ces pontes, on ne tarde pas à en voir sortir une multitude de minuscules bestioles : ce sont les jeunes larves, les larvules, relativement bien différentes des larves âgées; leurs membres sont grêles avec des poils démesurément longs; de simples tubes cylindriques nus représentent les branchies. C'est un premier, mais frappant exemple des différences de forme entre larves adultes ou âgées et larves naissantes (néonates) et les mœurs aussi diffèrent, car ces petites larves ne sont pas fouisseuses, mais nageuses évoluant en tous sens, ne fuyant pas la lumière et comme " planctoniques ". LESTAGE, après les anciens naturalistes, a figuré ces larvules et nous-mêmes avons pu compléter divers détails de la morphologie de la larvule des Sialis.

En Europe, les Mégaloptères ne sont représentés que par le genre Sialis ne comptant qu'un petit nombre d'insectes; ces insectes sont beaucoup plus nombreux en Amérique du Nord. En Amérique du Nord aussi, et encore dans diverses parties du monde, on rencontre des Mégaloptères dont les larves sont assez différentes, l'abdomen se terminant par des 


\section{Mégaloptères et Planipennes}

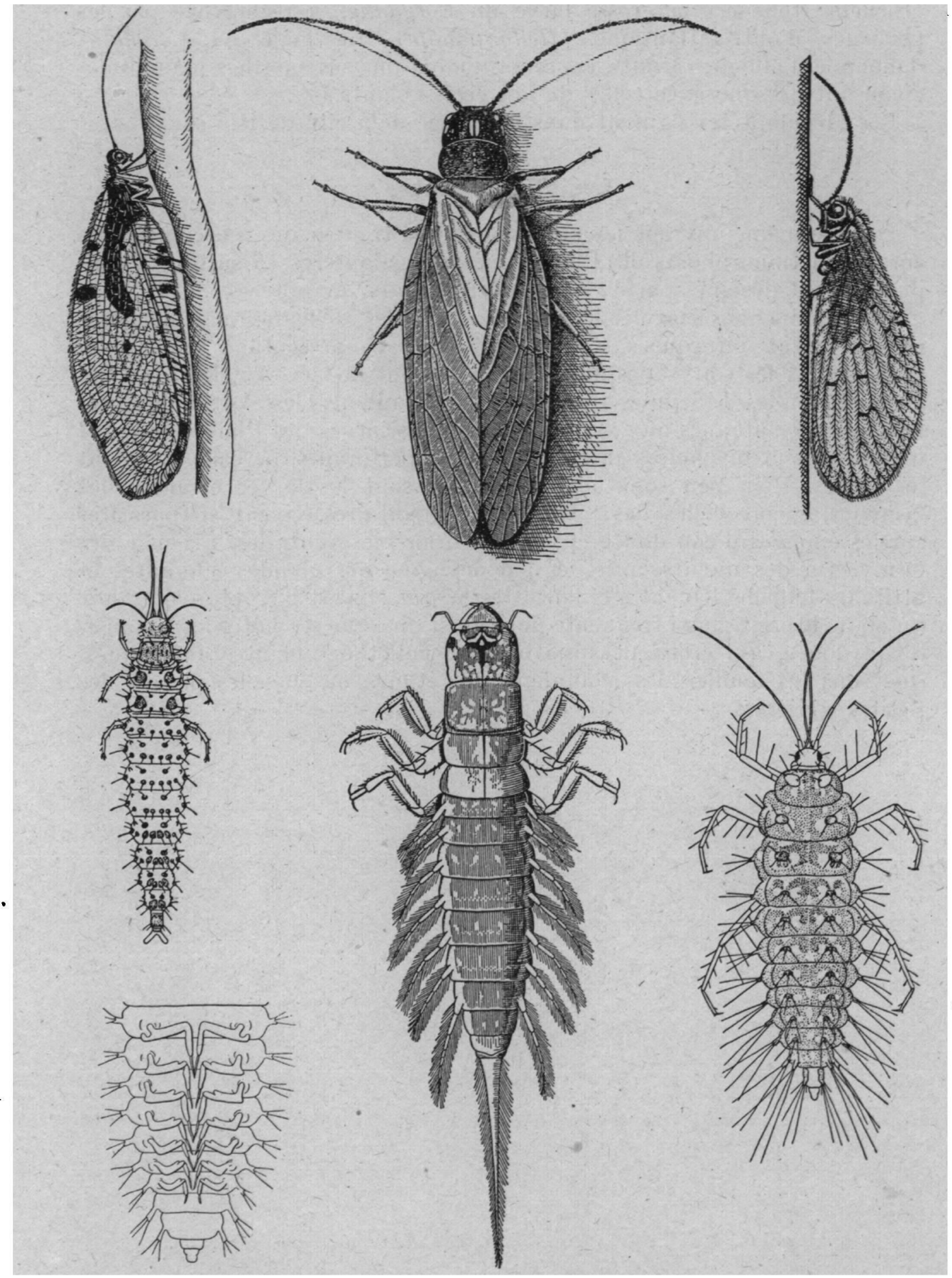

De gauche à droite et de haut en bas: Osmylus /ulvicephalus Scop.; Sialis lutaria L. ; Sisyra dalii MlcL. (d'après KIмм1кs); larve d'Osmylus fuloicephalus Scop. (d'après Rousszav); larve de Sialis fuliginosa Pict. (d'après Kum Ins); larve de Sisyra fuscata, face ventrale de l'abdomen de la méme, branohies (d'après Rousseav). 
crochets; telle est la grosse larve du Corydalis, si recherchée par les pêcheurs d'outre-atlantique (Hellgrammite). Le mâle du Corydalis, dénommé d'ailleurs cornuta, est remarquable par ses énormes mandibules rivalisant presque avec celles de nos cerfs-volants.

Les Mégaloptères figurent dans l'ouvrage déjà cité de Rousseau.

\section{Les Planipennes.}

Dans le mẻme ouvrage nous pouvons aussi trouver des renseignements sur les métamorphoses des Planipennes. Mégaloptères et surtout Planipennes sont désignés parfois sous le terme de "Névroptères".

Les Planipennes sont des insectes à ailes frêles, délicates, avec nervures régulièrement bifurquées. Certains de leurs représentants sont bien connus, tels les Chrysopes aux yeux d'or dont la larve est le "Lion des Pucerons ", les Libellules de nuit ou Fourmilions, les Ascalaphes, ces Libellules à antennes aux vives couleurs. Les larves des Planipennes ont mandibules et mâchoires en crocs grêles et perforants, perçant et suçant les proies. Bien peu sont aquatiques; ce sont les larves errantes des Osmyles, encore celles des Sisyres qui, sédentaires, vivent en parasites sur les éponges d'eau douce et ont des branchies ventrales. Le mâle des Osmyles a des mours curieuses : il dévagine des glandes odorantes et attire les femelles. Quant à la larve des Sisyra, elle est bien plus commune qu'on ne le croit, aussi fréquente pour ainsi dire que ses hôtes les Éponges d'eau douce, qui croissent aussi bien au collet des plantes aquatiques, que sous les feuilles des nénuphars des étangs ou sous les pierres des petites rivières. 